Original Research Paper

\title{
Determination of 1-Deoxynojirimycin Content and Phytochemical Profiles from Young and Mature Mulberry Leaves of Morus Spp.
}

\author{
Yasinta Ratna Esti Wulandari, Vivitri Dewi Prasasty, Adrianus Rio and Cindy Geniola \\ Faculty of Biotechnology, Atma Jaya Catholic University, Jakarta, Indonesia
}

\author{
Article history \\ Received: 13-04-2019 \\ Revised: 21-05-2019 \\ Accepted: 01-06-2019 \\ Corresponding Author: \\ Yasinta Ratna Esti Wulandari \\ Faculty of Biotechnology, \\ Atma Jaya Catholic University, \\ Jakarta, Indonesia \\ Email: yasinta.ratnaew@gmail.com
}

\begin{abstract}
Mulberry leaves have been known to be rich in 1-Deoxynojirimycin (DNJ) which inhibits postprandial hyperglycemia by inhibiting $\alpha$-glucosidase in the small intestine and thereby reducing the breakdown of starch and other complex sugar into glucose. Mulberry leaf extract also contains many other bioactive compounds and phytochemicals. They are very effective in supporting blood sugar levels and balancing cholesterol and triglycerides. The DNJ content in mulberry leaves depends on the leaf's variety and age. In this study, the phytochemical profile and DNJ content of young leaves and mature mulberry leaves in Indonesia were determined to further promote mulberry use using High Performance Liquid Chromatography (HPLC) and Pyrolisis Gass Chromatography-Mass Spectrometry (GC-MS). The phytochemicals test result for the four leaf samples showed that all four samples contain alkaloids, phenolics, flavonoids, tannins and terpenes. The mature leaves of $M$. cathayana contain the highest DNJ concentration $(2.90 \mathrm{mg} \mathrm{DNJ} / \mathrm{g}$ leaves) out of eight samples. DNJ has the potential to be used as a functional food for diabetic patients. Analysis using HPLC and Py-GC-MS confirmed that mulberry species contain many beneficial phytochemicals. This study presented that Indonesian mulberry leaves extracts to have high phytochemical compositions which very potential utilized in the medicinal field.
\end{abstract}

Keywords: 1-deoxynojirimycin, Mulberry, Morus spp., GC-MS, HPLC

\section{Introduction}

Mulberry is a plant from Moraceae family and Morus genus. Mulberry can grow in a wide variety of climate, from temperate to tropical, so this plant is cultivated in many regions, including Indonesia. Outside from its use as silkworm's feeds, mulberry leaves also used in traditional medicine because of its therapeutic effect (Ozgen et al., 2009) and pharmacology benefit (Song et al., 2009; Zafar et al., 2013).

In the medicinal field, many countries in Asia use mulberry leaves as a treatment for diabetes. The antidiabetic activity of the mulberry leaves already verified through studies with animal experiments and human trials (Hunyadi et al., 2013). This activity comes from various bioactive constituents inside the mulberry leaves, with the one that contributes most for anti-diabetic effect is 1deoxynojirimycin (DNJ) (Kimura et al., 2007).

DNJ is an alkaloid iminosugar, an analogue of glucose with -NH group substituting the oxygen atom on the pyranose ring. DNJ showed a high $\alpha$-glucosidase inhibitory activity; therefore, it can be used to control the postprandial blood glucose level on consumption ( $\mathrm{Hu}$ et al., 2013; Liu et al., 2012). Beside the anti-diabetic effect, DNJ was also reported to have antioxidant, antimicrobial and anti-inflammatory activities (Yin et al., 2010).

Based on previous studies, it is found that DNJ content in mulberry leaves may vary in different species. The DNJ content also affected by other factors such as the age of the leaves and the growth environment ( $\mathrm{Hu}$ et al., 2013; Yatsunami et al., 2008). Knowing this fact, this study aimed to further promote the use of mulberry leaves in Indonesia by determining DNJ content and the phytochemical profile of the mulberry leaves of Morus alba var. kanva-2, M. alba var. multicaulis, $M$. bombycis var. lembang and $M$. cathayana that was cultivated in Indonesia.

\section{Materials and Methods}

\section{Plant Material}

The mulberry leaf samples were collected from the University Farm of IPB University, Sukamantri, Bogor, 
Indonesia. A total of eight samples from four mulberry varieties were selected. The samples were the young leaves and mature leaves of $M$. alba var. kanva-2, $M$. alba var. multicaulis, M. bombycis var. lembang and $M$. cathayana. The young and mature leaves were classified based on branch position. The first leaves and the fifth leaves from branch apex were classified as young leaves and mature leaves respectively. All leaf sample was lyophilized and ground to a powder before analysis.

\section{Sample Extraction}

Procedures of sample extraction followed the description by $\mathrm{Hu}$ et al. (2013) and Vichasilp et al., (2009). Ninety milligrams of each sample was mixed with $2.5 \mathrm{~mL} 0.05 \mathrm{M} \mathrm{HCl}$, followed by sonication using ultrasonicator at $180 \mathrm{~W}$ and $40.000 \mathrm{~Hz}$ for $10 \mathrm{~min}$. The solutions then centrifuged at $17.000 \mathrm{~g}$ for 15 minutes. Initial supernatants were collected and the residues were re-extracted as described above. Both supernatants then pooled and diluted with distilled water to a final volume of $6 \mathrm{~mL}$.

\section{Preparation of DNJ Stock Solution}

Five milligrams of standard DNJ (Sigma Aldrich, USA) was mixed with $500 \mu \mathrm{L}$ distilled water to obtain the DNJ solution of $10 \mathrm{~g} / \mathrm{L}$. Seventy-five microliters of this solution was then added to $5 \mathrm{~mL} 0.05 \mathrm{M} \mathrm{HCl}$ and diluted with distilled water to a final volume of $10 \mathrm{~mL}$. DNJ concentration of the stock solution was $75 \mathrm{mg} / \mathrm{L}$. The stock solution was stored at $4^{\circ} \mathrm{C}$ until further use.

\section{Derivatization of $D N J$}

Procedures for DNJ derivatization conducted by Kim et al. (2003). Ten microliters of extract sample of DNJ standard solution was mixed with $10 \mu \mathrm{L} 0.4 \mathrm{M}$ potassium borate buffer ( $\mathrm{pH}$ 8). Twenty microliters of 9Fluoroenylmethyl Chloroformate (FMOC-Cl) $5 \mathrm{mM}$ in acetonitrile was added, the solution then vortexed for 30 seconds and incubated at $25^{\circ} \mathrm{C}$ for $20 \mathrm{~min}$. After that, $10 \mu \mathrm{L}$ $0.1 \mathrm{M}$ glycine was added to stop the reaction. The solution was diluted with $950 \mu \mathrm{L} 0.1 \%$ acetic acid to stabilize DNJFMOC and filtered using nylon syringe filter $(0.45 \mu \mathrm{m})$. Ten microliters of the filtrate was injected to HPLC to determine the DNJ concentration.

\section{Preparation of Standard Solution}

DNJ stock solution was derivatized according to the method described in DNJ derivatization. The derivatized stock solution was then diluted with distilled water to make standard solutions with a concentration of $2.28,4.56,9.13,18.25,37.5$ and 75 $\mathrm{mg} / \mathrm{L}$. The standard solutions were analyzed by HPLC and the relationship between peak area on chromatogram with DNJ concentration was used to construct a standard curve (Hu et al., 2011).

\section{Qualitative Phytochemical Tests}

The phytochemicals tested for its presence were alkaloids, phenolics, flavonoids, tannins and terpenes. For those tests, four drops of each sample were added with reagents corresponding to each tests on the spot plate.

\section{HPLC Analysis}

The derivatized extracts of all eight samples and standard solutions were analyzed with HPLC to determine their DNJ content. HPLC analysis was done using acetonitrile-acetic acid $0.1 \%(\mathrm{v} / \mathrm{v})$ as the mobile phase with $1: 1$ ratio at $1.0 \mathrm{~mL} /$ minutes elution rate for 14 minutes, silica C18 column at ambient temperature as stationary phase and VWD detector set at $254 \mathrm{~nm}$. After each analysis, the column was washed using methanol for 16 minutes to remove impurities on the column and equilibrated with mobile phase for $10 \mathrm{~min}$ (Kim et al., 2003). All the chemicals used in the HPLC analysis were of HPLC grade and the water used was double distilled. The HPLC analysis of each sample was done in triplicate. The data presented were the mean \pm Standard Deviation (SD).

\section{Py-GC-MS Analysis}

Ground leaf samples were analyzed using Py-GC-MS instrument QP2010 (Shimadzu, Duisburg). The stationary phase was capillary column (Phase Rtx-5MS) $60 \mathrm{~m} \times 0.25$ $\mathrm{mm}$ ID and the mobile phase was helium gas. Pyrolysis temperature was set at $400^{\circ} \mathrm{C}$ (Nandagopal et al., 2014).

\section{Results and Discussion}

\section{Qualitative Phytochemical Tests}

The result of the qualitative phytochemical tests of eight mulberry leaf samples is presented in Table 1 . This result revealed that alkaloids, phenolics, flavonoids, tannins and terpenes are present in all eight leaf samples.

Phytochemicals are bioactive compounds that mainly found in plants. Phytochemicals can be divided into a few major groups, which are alkaloids, phenolics and terpenes (Niratker et al., 2014). The result of preliminary qualitative tests in this study shows that all mulberry leaf samples contain all major groups of phytochemicals, which are alkaloids, phenolics, flavonoids, tannins and terpenes. The phytochemicals in the mulberry leaves are responsible for its therapeutic effect in the medicinal field.

In the plant, terpenoid compound used to attract insect (Wadood et al., 2013). From recent studies, it was discovered that this compound mostly responsible for this effect is some iminosugars and phenolics compounds (Hunyadi et al., 2013; Liu et al., 2012). These various phytochemicals contain in the mulberry leaves can be further studied to determine its physiological actions on the human and can be seen as a potential source of useful drugs. 
Table 1: Phytochemical test results of leaf samples

\begin{tabular}{|c|c|c|c|c|c|c|c|c|}
\hline \multirow[b]{2}{*}{ Phytochemicals } & \multicolumn{2}{|c|}{ M. alba var. kanva-2 } & \multicolumn{2}{|c|}{ M. alba var. multicaulis } & \multicolumn{2}{|c|}{ M. bombycis var. lembang } & \multicolumn{2}{|c|}{ M. cathayana } \\
\hline & Young & Mature & Young & Mature & Young & Mature & Young & Mature \\
\hline A. Alkaloids & & & & & & & & \\
\hline Wagner's test & + & + & + & + & + & + & + & + \\
\hline $\begin{array}{l}\text { Mayer's test } \\
\text { B. Phenolics }\end{array}$ & + & + & + & + & + & + & + & + \\
\hline $\begin{array}{l}\mathrm{FeCl}_{3} \\
\text { C. Flavonoids }\end{array}$ & + & + & + & + & + & + & + & + \\
\hline $\begin{array}{l}\mathrm{NaOH} \text { test } \\
\text { D. Tannins }\end{array}$ & + & + & + & + & + & + & + & + \\
\hline $\begin{array}{l}\text { Pb-acetate test } \\
\text { E. Terpenes }\end{array}$ & + & + & + & + & + & + & + & + \\
\hline Liebermann-burchard's test & + & + & + & + & + & + & + & + \\
\hline
\end{tabular}

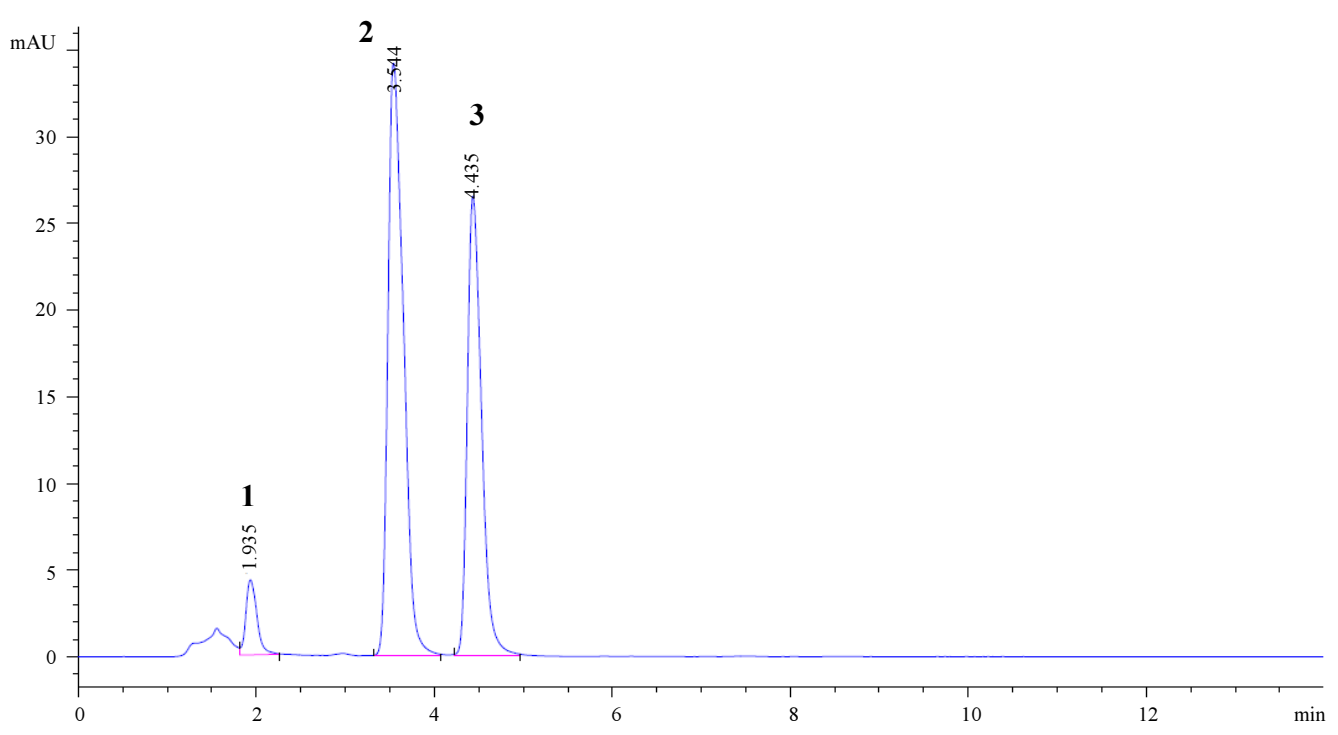

Fig. 1: Chromatogram standard curve. Number 1 represents FMOC-OH; 2) FMOC-DNJ; 3) FMOC-Gly

DNJ is one of the iminosugars found in the mulberry leaves. Many of the iminosugars show inhibitory activity against glucosidases, so they have been receiving great attention in the pharmaceutical industry. DNJ is a potent $\alpha-$ glucosidase inhibitor while also having antioxidant, antimicrobial and anti-inflammatory activity (Hu et al., 2013). It has an excellent $\alpha$-glucosidase inhibitory activity in vitro and is suitable to use as a functional food to treat diabetic patients (Kim et al., 2003). Thus, it is necessary to find a good source of DNJ since DNJ has high potential to overcome diabetic.

\section{HPLC Analysis}

The standard solutions were analyzed by HPLC and the results obtained were used to construct a standard curve (Fig. 1). The equation from the standard curve was then used to calculate the actual DNJ concentration in the leaf samples.
The chromatogram from HPLC analysis of leaf samples is shown on Fig. 2. The DNJ retention time is around $3.5 \mathrm{~min}$.

The result of DNJ determination by using HPLC is presented in Table 2. The result showed that the highest DNJ content is in mature $M$. cathayana leaves. The result also showed that the mature leaves of all samples contain higher DNJ concentration.

The mulberry leaves are known to have high DNJ content. From the eight leaf samples analyzed, the DNJ content greatly varies between leaf samples. This is because the mulberry varieties and the age of mulberry leaves could affect DNJ concentration (Hu et al., 2013; 2011). In this study, $M$. cathayana leaves have the highest DNJ content over all sample with a concentration of DNJ in the mature leaves of M. cathayana contain DNJ as high as $2.90 \mathrm{mg}$ $\mathrm{DNJ} / \mathrm{g}$ leaves. For comparison, the DNJ content of the mulberry leaves also has been determined in other studies. 
Table 2: DNJ determination of leaf samplesA

\begin{tabular}{ll}
\hline Leaf samples & DNJ Concentration $(\mathrm{mg}$ DNJ/g leaves) \pm SD \\
\hline Morus alba var. kanva-2 & \\
Young leaves & $0.07 \pm 0.03$ \\
Mature leaves & $0.27 \pm 0.08$ \\
M. alba var. multicaulis & \\
Young leaves & $0.077 \pm 0.018$ \\
Mature leaves & $0.157 \pm 0.058$ \\
M. bombycis var. lembang & \\
Young leaves & $0.136 \pm 0.039$ \\
Mature leaves & $1.413 \pm 0.023$ \\
Morus cathayana & \\
Young leaves & $0.94 \pm 0.11$ \\
Mature leaves & $2.90 \pm 0.57$ \\
\hline
\end{tabular}

The studies on China mulberry varieties got the DNJ content ranging from $0.10-1.60 \mathrm{mg} \mathrm{DNJ} / \mathrm{g}$ leaves (Hu et al., 2013), while studies on Thailand mulberry varieties were found DNJ content ranging from $0.30-3.20 \mathrm{mg} \mathrm{DNJ} / \mathrm{g}$ leaves (Vichasilp et al., 2009). This makes the DNJ content of mature $M$. cathayana is quite high compared to the DNJ content of another mulberry varieties. All of the mature leaves also have higher DNJ content young leaves, which differ with another study that found that DNJ content is higher in young leaves than in mature leaves $(\mathrm{Hu}$ et al., 2011). This is possible because of the difference in variety and growth environment of the mulberry. However, the definite biosynthesis pathway of DNJ is still not known (Yatsunami et al., 2008).

HPLC chromatograms showed a different pattern for the young and mature leaves from all species in this study. This means that the nitrogen-containing compound from the young and mature leaves of those species are different since the FMOC derivatizes the primary and secondary amine containing a compound in the samples (Kim et al., 2003). Interestingly, some other peaks arose in particular both $M$. bombycis var. lembang young and mature leaves on Fig. 2E and $2 \mathrm{~F}$. These peaks might represent the other constituents such as limonene, benzoic acids, flavonoid derivatives, anthocyanins and polyphenols. Mulberry leaves are believed can be used as potential food materials due to their nutritional and functional components (Yu et al., 2018). Mulberry is also known to have fatty acid (linolenic acid, palmitic acid, oleic acid) content. Moreover, many volatile constituents also found in mulberry, such as acetic acid, ethyl butyrate, 3-hydroxyl-2-butanone, ethyl acetate, 3methyl butanal, 2-methyl butanal, heptanal, hexanal, methional, trans-2-hexanal, 2-octenone, hexanoic acid, benzaldehyde, methyl hexanoate, 2-ethylhexanal, octanal, ethyl hexanoate, limonene and ethyl decanoate (Zhang et al., 2018). All HPLC chromatogram showed the highest peak on retention time at 4.4 minutes, where this peak was presumed to belong l-limonene.
These constituent compositions may vary according to different ecological conditions (Yu et al., 2018).

\section{Py-GC-MS Analysis}

The analysis of leaf samples using Py-GC-MS gives a pyrogram which detect many organic compounds. From all the organic compounds detected, the phytochemicals that were found are listed in Table 3. All the phytochemicals were in terpenes group. Mature leaves of all samples give a wider variety and higher amount of terpenes with this analysis.

Py-GC-MS is a powerful technique to analyze the component of the samples. It only needs a minute amount of sample and the sample does not need to be derivatized before analysis. With the pyrolysis, the sample component will either flash vaporized if the molecule is volatile and thermally stable or degraded into lighter molecules if the molecule is large and hard to volatilize (Montalbani, 2012). The pyrolysis of the eight leaf samples were done to see whether the phytochemicals in the samples can be detected by this method. The results showed that only a few simple terpenes on each sample were successfully analyzed. The simple terpene is easier to volatilize because it is lack of polar group, while the other phytochemicals like alkaloids and phenolics mostly contain amide and hydroxyl group respectively, making them more polar and harder to volatilize (Kusch, 2012). The other phytochemicals present in the sample are degraded and detected as a simpler organic molecule in the pyrogram, so it is not possible to analyze the whole phytochemicals content of the mulberry leaf samples using this technique. The terpenes found on the leaf sample pyrograms cannot be treated as total terpenes contents since it is unknown if all the terpenes are successfully volatilized or not. The volatile terpenes detected in the mulberry leaves like chamigrene and farnesene may play a role in the leaves defense mechanism against the pathogen (Courtois et al., 2012). But, some of the terpene fractions found in the sample have a beneficial effect. The phytol is known to have antimicrobial properties as phytomedicine (Rajab et al., 1998) and the limonene is known to have anticancer properties (Zheng et al., 1992). 

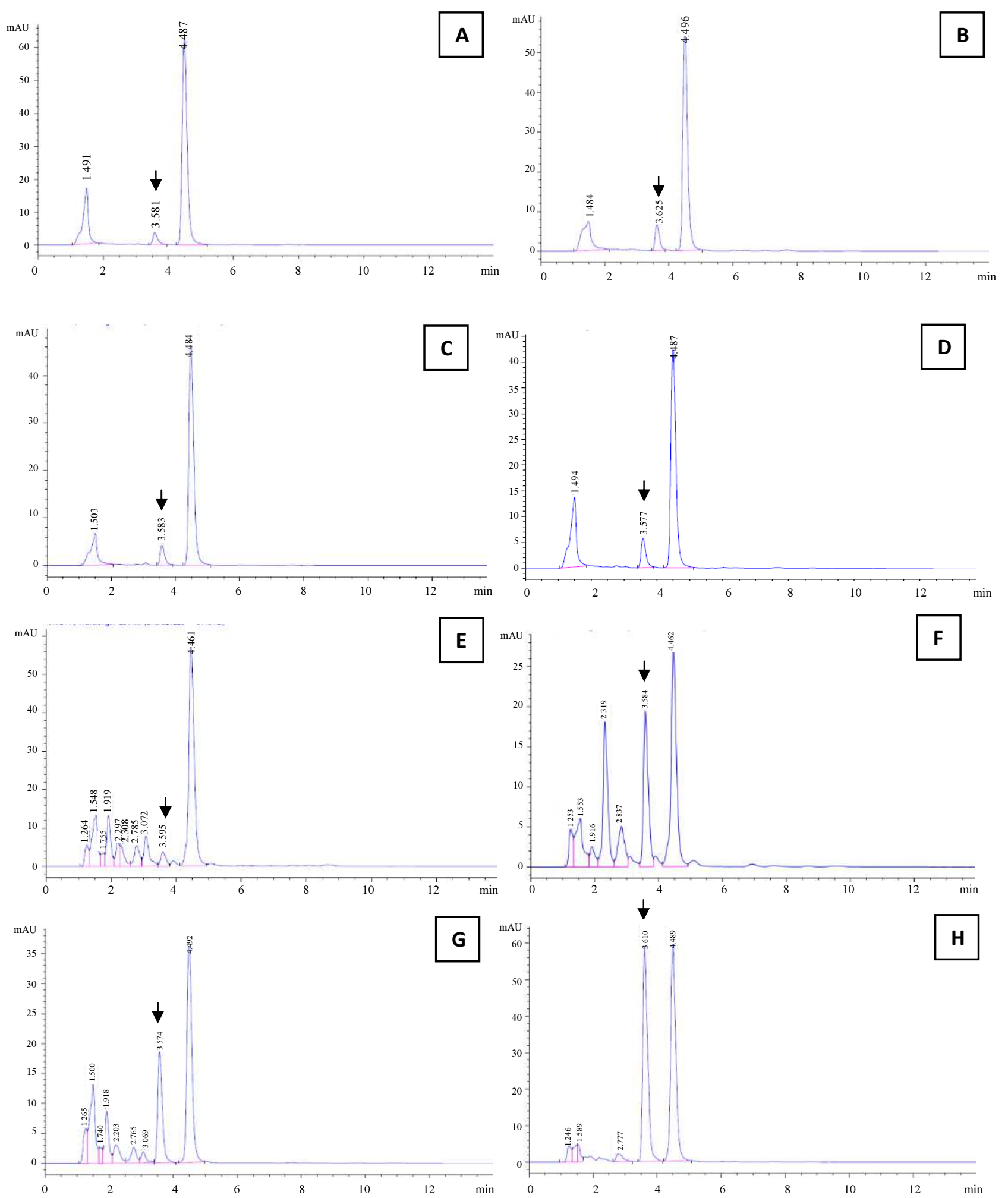

Fig. 2: Chromatogram of leaf samples: (A) M. alba var. kanva-2 young leaves (B) M. alba var. kanva-2 mature leaves (C) M. alba var. multicaulis young leaves (D) M. alba var. multicaulis mature leaves (E) M. bombycis var. lembang young leaves (F) $M$. bombycis var. lembang mature leaves $(\mathrm{G})$ M. cathayana young leaves (H) M. cathayana mature leaves. DNJ 's peaks showed by the arrow 
Table 3: Secondary metabolite constituents from Py-GC-MS analysis

\begin{tabular}{llll}
\hline Leaf samples & Secondary metabolites & Class & Concentration \\
\hline Young $M$. alba var. kanva- & Limonene & Monoterpenes & $1.12 \%$ \\
Mature $M$. alba var. kanva-2 & Phytol & Diterpenes & $0.69 \%$ \\
& Phytol & Diterpenes & $8.16 \%$ \\
& Limonene & Monoterpenes & $6.33 \%$ \\
Young $M$. alba var. multicaulis & Citronella & Monoterpenes & $1.87 \%$ \\
& Neophytadiene & Sesquiterpenes & $4.52 \%$ \\
Mature $M$. alba var. multicaulis & Limonene & Monoterpenes & $13.53 \%$ \\
Young $M$. bombycis var. lembang & Dihidrocarveol & Monoterpenes & $0.65 \%$ \\
Mature $M$. bombycis var. lembang & Limonene & Monoterpenes & $5.58 \%$ \\
& Cycloundecene & Sesquiterpenes & $0.44 \%$ \\
Young $M$. cathayana & Neophytadiene & Sesquiterpenes & $3.81 \%$ \\
Mature $M$ cathayana & N/A* & Monoterpenes & $14.24 \%$ \\
& Limonene & Diterpenes & $3.24 \%$ \\
& Chytol & Sesquiterpenes & $0.81 \%$
\end{tabular}

*Not Available

However, the root rot may threat the mulberry plant. It has been reported that Macrophomina phaseolina (Tassi) Goid causes charcoal rot which has the predominant pathogen to which all productive mulberry cultivars. Thus, controlling the quality of mulberry plant may maintain the phytochemical constituents in mulberry (Pinto et al., 2018). Limonene also detected in $M$. bombycis var. lembang mature leaves, 1-limonene and cyclododecane are secondary metabolites are known to have antimicrobe, antiviral, immunosuppressant, antiallergic, skincare and trigger hair growth (Nahbar et al., 2012). Previous studies showed that mulberry plants have potential pharmacological properties as antidiabetes by naturally inhibiting $\alpha$-glucosidase activity. Liu et al. (2015) demonstrated that mulberry leave extracts presented $\mathrm{Ki}$ value was $56.53 \mathrm{mg} / \mathrm{mol}$ as a competitive inhibition mode against $\alpha$-glucosidase. However, the other parts of mulberry are also have potential antidiabetic effect as well. Thus, it suggested that mulberry plant is a good nutritional source as natural antidiabetic agent (Liu et al., 2015).

\section{Conclusion}

In the course of the research, all samples contain various phytochemicals, including alkaloids, phenolics, flavonoids, tannins and terpenes that can be beneficial to our health, which DNJ as one of them.

DNJ is known to have an antidiabetic effect. The mature leaves of $M$. cathayana contain the highest DNJ of all the eight samples used ( $2.90 \mathrm{mg} \mathrm{DNJ} / \mathrm{g}$ leaves) and have the potential to be used as functional foods for diabetic patients. The mulberry leaves also still contain many beneficial phytochemicals to be further discovered.

\section{Acknowledgment}

The author like to express the deepest appreciation to University Farm of IPB University, Sukamantri, Bogor, Indonesia, to provide the plant materials.

\section{Funding Information}

This project was fully funded by the Faculty of Biotechnology, Atma Jaya Catholic University of Indonesia.

\section{Author's Contributions}

All authors participated in all experiments, coordinated the data-analysis and contributed to the writing of the manuscript.

\section{Ethics}

This article is original and contains unpublished material. The corresponding author confirms that all of the other authors have read and approved the manuscript and no ethical issues involved.

\section{References}

Courtois, E.A., C. Baraloto, C.E. Paine, P. Petronelli and P.A. Blandinieres et al., 2012. Differences in volatile terpene composition between the bark and leaves of tropical tree species. Phytochemistry, 82: 81-88. DOI: 10.1016/j.phytochem.2012.07.003.

Hu, K., Y. Li, Y. Du, B. Su and D. Lu, 2011. Analysis of 1-deoxynojirimycin component correlation between medicinal parasitic loranthus from loranthaceae and their mulberry host trees. J. Med. Plants Res., 5: 4326-4331. ISSN: 1996-0875. 
Hu, X.Q., L. Jiang, J.G. Zhang, W. Deng and H.L. Wang et al., 2013. Quantitative determination of 1deoxynojirimycin in mulberry leaves from 132 varieties. Industrial Crops Products, 49: 782-784. DOI: $10.1016 /$ j.indcrop.2013.06.030.

Hunyadi, A., E.L. Busa, A. Marki, A. Martins and N. Jedlinszki et al., 2013. Metabolic effects of mulberry leaves: Exploring potential benefits in type 2 diabetes and hyperuricemia. Evidence-Based Complementary Alternative Medicine, 2013: 948627-948627. DOI: 10.1155/2013/948627.

Kim, J.W., S.O. Kim, H.S. Lee, I. Kim and M.Y. Ahn et al., 2003. Determination of 1-deoxynojirimycin in Morus alba L. leaves by derivatization with 9fluorenylmethyl chloroformate followed by reversed-phase high-performance liquid chromatography. J. Chromatography, 1002: 93-99. DOI: 10.1016/S0021-9673(03)00728-3.

Kimura, T., K. Nakagawa, H. Kubota, Y. Kojima and Y. Goto et al., 2007. Food-grade mulberry powder enriched with 1-deoxynojirimycin suppresses the elevation of postprandial blood glucose in humans. J. Agric. Food Chem., 55: 5869-5874.

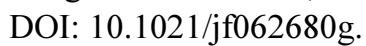

Kusch, P., 2012. Pyrolysis-Gas Chromatography/Mass Spectrometry of Polymeric Materials. In: Advanced Gas Chromatography-Progress in Agricultural, Biomedical and Industrial Applications, Mohd, M.A., (Eds.), Tech, Rijeka.

Liu, C., W. Xiang, Y. Yu, Z.Q. Shi and X.Z. Huang et al., 2015. Comparative analysis of 1deoxynojirimycin contribution degree to $\alpha$ glucosidase inhibitory activity and physiological distribution in Morus alba L. Industrial Crops Products, 70: 309-315.

DOI: 10.1016/j.indcrop.2015.02.046

Liu, M., S. Wang, Y.D. Zhou, T. Xiang and H. Dong et al., 2012. An iminosugar N-pentafluorobenzyl-1deoxynojirimycin as a novel potential immunosuppressant for the treatment of Th2-related disease. Bioorganic Med. Chem. Lett., 22: 564-570. DOI: $10.1016 /$ j.bmcl.2011.10.081.

Montalbani, S., 2012. Pyrolysis-gas chromatographymass spectrometry and chemometric analysis for the characterization of complex matrices [Thesis]. University of Bologna, Bologna.

Nahbar, N., A. Shafagnat and F. Salimi, 2012. Antimicrobial activity and constituents of the hexane extracts from leaf and stem of Origanum vulgare L. ssp. Viride (Boiss.) Hayek growing wild in Northwest Iran. J. Med. Plants Res., 6: 2681-2685. DOI: $10.5897 /$ JMPR11.1768.
Nandagopal, S., A.G. Kumar, D.P. Dhanalaksmi and P. Prakash, 2014. Bio-Prospecting the antibacterial and anticancer activities of silver nanoparticles synthesized using Terminalia chebula seed extract. Int. J. Pharmacy Pharmaceutical Sci., 6: 368-373. DOI: $10.1007 / \mathrm{s} 13205-014-0197-x$.

Niratker, C., Preeti and M. Singh, 2014. Preliminary phytochemical screening of leaf extract of Mulberry (Morus indica) from Chhattisgarh. Int. J. Applied Biol. Pharmaceutical Technol., 5: 131-136. ISSN: 0976-4550.

Ozgen, M., S. Serce and C. Kaya, 2009. Phytochemical and antioxidant properties of anthocyanin-rich Morus nigra and Morus rubra fruits. Sci. Horticulturae, 119: 275-279.

DOI: 10.1016/j.scienta.2008.08.007.

Pinto, M.V., H.S. Poornima, M.S. Rukmangada, R. Triveni and V.G. Naik, 2018. Association mapping of quantitative resistance to charcoal root rot in mulberry germplasm. PloS one, 13: e0200099.

Rajab, M.S., C.L. Cantrell, S.G. Franzblau and N.H. Fischer, 1998. Antimycobacterial activity of (E)phytol and derivatives: A preliminary structureactivity study. Planta Med., 64: 2-4.

Song, W., H.J. Wang., P., Bucheli, P.F. Zhang, D.Z. Wei and Y.H. Lu, 2009. Phytochemical profiles of different Mulberry (Morus sp.) species from China. J. Agric. Food Chem., 57: 9133-9140.

DOI: $10.1021 / \mathrm{jf9022228.}$

Vichasilp, C., K. Nakagawa, P. Sookwong, Y. Suzuki and F. Kimjura et al., 2009. Optimization of 1deoxynojirimycin extraction from mulberry leaves by using response surface methodology. Biosci. Biotechnol. Biochem., 73: 2684-2689.

DOI: $10.1271 / \mathrm{bbb} .90543$.

Wadood, A., M. Ghufran, S.B. Jamal, M. Naeem and A. Khan et al., 2013. Phytochemical analysis of medicinal plants occuring in local area of Mardan. Biochem. Analytical Biochem., 2: 144-144. DOI: $10.4172 / 21611009.1000144$.

Yatsunami, K., M. Ichida and S. Onodera, 2008. The relationship between 1-deoxynojirimycin content and $\alpha$-glucosidase inhibitory activity in leaves of 276 mulberry cultivars (Morus spp.) in Kyoto, Japan. J. Natural Med., 62: 63-66. DOI: $10.1007 / \mathrm{s} 1141800701850$.

Yin, H., X.Q. Shi, B. Sun, J.J. Ye and Z.A. Duan et al., 2010. Accumulation of 1-deoxynojirimycin in silkworm, Bombyx mori L. J. Zhejiang University Sci. B, 11: 286-291. DOI: 10.1631/jzus.B0900344.

Yu, Y., H. Li, B. Zhang, J. Wang and X. Shi et al., 2018. Nutritional and functional components of mulberry leaves from different varieties: Evaluation of their potential as food materials. Int. J. Food Properties, 21: 1495-1507. 
Zafar, M.S., D. Muhammad, I. Javed, M. Akhtar and T. Khaliq et al., 2013. White mulberry (Morus alba): A brief phytochemical and pharmacological evaluations account. Int. J. Agric. Biol., 15: 612-620.

Zhang, H., Z. Ma, X. Luo and X. Li, 2018. Effects of mulberry fruit (Morus alba L.) consumption on health outcomes: A mini-review. Antioxidants, 7: 1-13.
Zheng, G.Q., P.M. Kenney and L.K. Lam, 1992. Anethofuran, carvone and limonene: Potential cancer chemopreventive agents from dill weed oil and caraway oil. Planta Med., 58: 338-341. 